\title{
Solvent-Containing Closure Material Can Be Used to Prevent Follicular Penetration of Caffeine and Fluorescein Sodium Salt on Porcine Ear Skin
}

\author{
Anna Lena Klein ${ }^{a}$ Markus Lubda ${ }^{b}$ Paniz Akbarzadeh Taghavi ${ }^{a}$ c \\ Jürgen Lademann ${ }^{a}$ Ingeborg Beckers ${ }^{c}$ Jörg von Hagen ${ }^{b}$ Harald Kolmar ${ }^{d}$ \\ Alexa Patzelt ${ }^{a}$ \\ ${ }^{a}$ Charité - Universitätsmedizin Berlin, corporate member of Freie Universität Berlin, Humboldt-Universität zu Berlin, \\ and Berlin Institute of Health, Department of Dermatology, Venereology, and Allergology, Center of Experimental

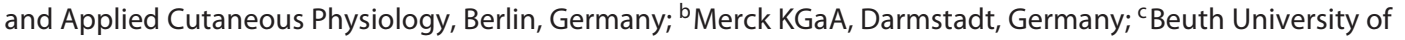 \\ Applied Sciences Berlin, Berlin, Germany; ${ }^{\mathrm{d}}$ Technische Universität Darmstadt, Darmstadt, Germany
}

\section{Keywords}

Follicular closing · Follicular penetration - Skin penetration · Laser scanning microscopy · Caffeine

\begin{abstract}
Aim: The skin represents a drug delivery portal. The establishment of a skin model capable of distinguishing between the follicular and intercellular penetration pathways remains a challenge. The study described herein was aimed at showing the influence of two nail varnishes as closure material and four application techniques to spread the active pharmaceutical ingredient (API) on a successful follicular closure without inducing penetration-enhancing effects. Materials and Methods: For all experiments, ex vivo porcine ear skin was used. In study design A, a standard and a solvent-free nail varnish were compared. It was tested whether the different application techniques (spreading with pipette, careful finger massage, $5-\mathrm{Hz}$ finger massage, $5-\mathrm{Hz}$ automatic massage) potentially destroy an intact follicular closure. Laser scanning microscopy imaging was used to measure if the model drug (fluorescein sodium salt) penetrated into the
\end{abstract}

\section{KARGER}

(c) 2020 S. Karger AG, Basel

karger@karger.com

www.karger.com/spp hair follicles. Study design B investigated how the penetration is affected when applying standard nail varnish containing solvents to skin. It was tested if the varnish blocks the API (caffeine) on completely covered areas and if adjacent areas show increased penetration. Furthermore, lateral diffusion of the API was investigated. After $20 \mathrm{~h}$, the skin layers were separated by tape stripping and heat separation. The tissue samples were homogenized. Caffeine was quantified by chromatography. Results: In study design A, the standard nail varnish showed a secure follicular closure, while the solvent-free nail varnish was not able to prevent follicular penetration. Moreover, rapid application techniques were found to destroy an intact follicular closure. Only the two most gentle application techniques kept the follicular closing intact. In study design B, no caffeine was detected in both skin areas that were completely covered. Since no significant difference in caffeine penetration between the two uncovered groups was found, any influence of the applied closure material on adjacent areas was excluded. Conclusion: This

Anna Lena Klein and Markus Lubda share first authorship. 
study clearly demonstrates that a standard nail varnish in combination with a gentle application technique of the API provides a secure follicular closure. The presented study only investigated the closure for the substances caffeine and fluorescein sodium salt. The results might not be transferable to all kinds of APIs.

(c) 2020 S. Karger AG, Basel

\section{Introduction}

The skin is the physical barrier to our environment [1] and accounts for approximately $16 \%$ of the human body weight, with a surface area of around $2 \mathrm{~m}^{2}$ in human adults [2]. Due to its strong barrier properties, the skin represents a challenging drug delivery portal, which requires appropriate technologies for successful dermal or transdermal drug delivery. There are three main delivery routes for a percutaneous permeation of targeted pharmacological molecules through this heterogeneous organ: (I) the intercellular, (II) the transcellular, and (III) the follicular penetration pathway. For follicular penetration, two distinct penetration routes are feasible, namely the intrafollicular and the transfollicular penetration pathway. While the intercellular penetration pathway has been investigated in detail, the role of follicular penetration for transdermal drug delivery has long been underestimated due to the small surface area of hair follicles on the skin $[3,4]$. As the hair follicles are heterogeneously distributed across the skin surface, shunt route penetration is different in various body regions. It is known that higher absorption rates are achieved on the forehead compared to the extremity (forearm and calf) regions [5]. The follicular epithelium can be seen as the enlargement of the skin surface with similar barrier properties at the upper portion of the hair follicle. However, the lower part is more permeable due to less differentiated corneocytes, which display reduced skin barrier properties compared to the interfollicular epithelium $[4,5]$.

The establishment of a skin model that is able to differentiate between the follicular and intercellular penetration pathway is a challenge. It is necessary to find a model system that safely excludes the follicular penetration shunt route but has the same structural, biochemical, and barrier properties as normal skin [6]. A relatively obvious approach is to compare skin penetration with different follicular densities or to compare hairy and hairless species $[7,8]$. The problem of this approach is that skin with different hair follicle densities may also have other structural properties. Moreover, a comparison of scarred skin [9] and normal skin is rather critical to evaluate, since the barrier properties of the skin are certainly not comparable.

The method of selective follicle closure has been developed to evaluate the impact of the follicular penetration pathway. In this approach, each hair follicle is closed selectively in the test area, whereas in the control area of the same skin probe, hair follicles are left open. A comparison of the percutaneous absorption rates of both skin areas can be used to calculate the proportion of the hair follicles to the total absorption [10].

As follicular closing material, different substances like nail varnish $[5,10,11]$, a silicone grease-cyanoacrylate adhesive mixture [12], waxes [13], and Teflon [10] have been proposed, with nail varnish being utilized in most of the studies. Since the use of nail varnish as a closing material in particular could have side effects such as penetration enhancement in the adjacent skin areas, the aim of the present study was, inter alia, to investigate this potential effect. Solvents like ethanol and acrylates, which are frequent ingredients of nail varnish, show a statistically enhanced deposition of topically applied substances in the stratum corneum [14] and are known to partially disrupt the skin barrier [15]. Due to this fact, the assessment of solvent-free closure material is of high interest and was included in our investigations.

It is known that topically applied substances do not only penetrate vertically into the skin but that a possible lateral diffusion can occur [16]. If the test substance were prone to strong lateral diffusion in the used formulation after penetration into the viable layers of the skin, the influence of the hair follicles on the penetration could not be excluded. To better understand this relationship, lateral diffusion was also considered in the experiments.

Moreover, the correct application protocol of the closing material on the follicular openings and the model drug of interest seems to play a major role for a successful sealing of the hair follicles and therefore, different options of application were assessed in this study.

The selection of an appropriate skin model has been demonstrated to have a major impact on the results of follicular penetration investigations. The method of selective follicular closure has been developed for in vivo studies in humans [10]. A transfer of the method to ex vivo skin models is difficult, as the hair follicles tend to contract vigorously in excised skin samples. In a former study, the follicular reservoir was shown to be reduced by $90 \%$ in excised human skin in comparison to in vivo skin [5]. As a consequence, the ex vivo porcine ear skin model has been established for follicular penetration studies, as 
Fig. 1. Schematic illustration and photograph of the follicular closure technique.

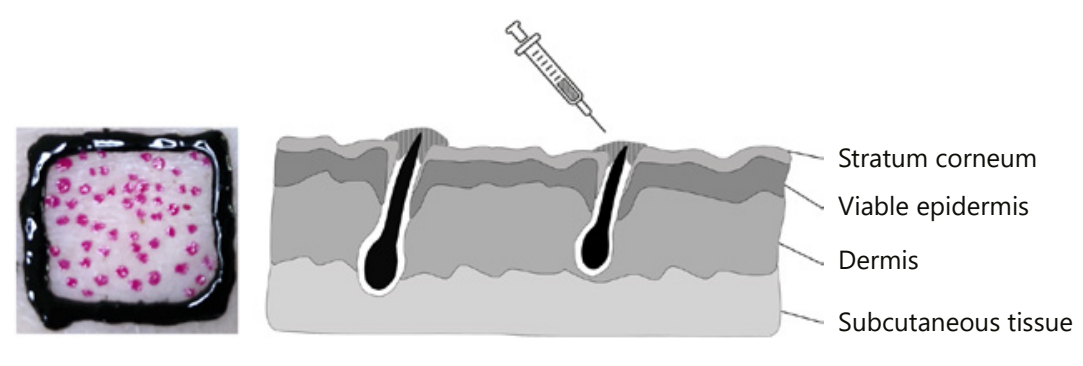

porcine skin remains fixed on the cartilage during the experiments and does not contract. Moreover, porcine ear skin provides similar morphological and barrier abilities and a comparable composition of corneocytes to human skin [17]. Furthermore, it has been proven to be a suitable surrogate for in vivo bioequivalence studies on human percutaneous penetration $[18,19]$.

Stahl et al. [20] also investigated the reliability of nail varnish used for follicular closure. In this study, a variety of test substances were used. The authors found out that the efficiency of the follicular closure depends on the investigated test substance. In the presented investigations, caffeine was used as active pharmaceutical ingredient (API). With its naturally occurring purine-based alkaloid structure, caffeine is widely used for penetration studies and recommended by the OECD in their guidelines for testing percutaneous absorption [21]. As caffeine has already been shown to penetrate faster and at a higher quantity if the follicular penetration pathway is available [5], caffeine was selected as active substance in the present study. In the following, caffeine is also referred to as API.

The aim of the present study was to establish a reliable protocol for the follicular closing technique to facilitate the investigation of the follicular penetration path. This closing technique was tested for its ability to prohibit the penetration of the applied test substances.

\section{Materials and Methods}

\section{Skin Model}

For the experiments, ears from 6-month-old German domestic pigs were provided by a local butcher (Gerald Nusche, Königs Wusterhausen, Germany) and stored at $8^{\circ} \mathrm{C}$ until use. The experiments were authorized by the Commission of Consumer Protection and Agriculture, District Dahme-Spreewald, Germany. Before starting the experiments, the ears were rinsed with cold water and dried with paper towels. The ex vivo porcine ear skin was used within 1 or 2 days after slaughtering. To produce a secure follicular closure by using one of the closure materials described below, the hairs had to be cut close to skin surface level by scissors to ensure that the tips of the hairs were completely covered by the closure material.

\section{Follicular Closing Technique}

In the present study, the hair follicles were closed either with a standard nail varnish $\left(\mathrm{N}^{\circ} 1\right.$ Nail Polish 290 , trend it up, dmdrogerie markt $\mathrm{GmbH} \& \mathrm{Co}$. KG) containing different alcohols, acrylates, and acetates or a solvent-free nail varnish with the filmforming agent Syntran ${ }^{\circledR}$. The hair follicles were plugged by placing dots of the closure material precisely on top of each follicular orifice by using a cannula (26 G, Dispomed Witt oHG, Gelnhausen, Germany) and a 1-mL syringe according to Teichmann et al. [10]. After a drying time of $30 \mathrm{~min}$, the model drug was applied. The method is schematically depicted in Figure 1.

Study Design

The study was divided into two main parts.

Study Design A: Comparison of the Efficiency of a Standard Nail Varnish and a Solvent-Free Nail Varnish for Selective

Hair Follicle Closure and Investigation of the Impact of the Application Protocol

Study design A involved two experimental setups, hereinafter referred to as A1 and A2. In setup A1, the follicular closing technique was performed with standard nail varnish or a solvent-free nail varnish, as described above. In total, twelve skin areas (a to l) were prepared. In four skin areas, all hair follicles were closed with a standard nail varnish (areas a-d) and in another four skin areas, all hair follicles were closed with a solvent-free nail varnish (areas $\mathrm{e}-\mathrm{h}$ ). Four skin areas remained untreated and served as control for each application technique (areas i-l). Each skin area had a diameter of $1.5 \times 1.5 \mathrm{~cm}$. To prevent spreading of the applied gel across the assigned limit of the area, black window color (Art-Glass, WACO, Heinrich Wagner, Fulda, Germany) was placed around the areas. Three hair follicles of each area were investigated.

After follicular closing, $10 \mu \mathrm{L} / \mathrm{cm}^{2}$ of a fluorescein gel ( $1 \%$ fluorescein sodium salt [Sigma-Aldrich, Steinheim, Germany] in $\mathrm{H}_{2} \mathrm{O}$ and 7\% hydroxyethyl cellulose [Euro OTC Pharma GmbH, Bönen, Germany], prepared $24 \mathrm{~h}$ before application) was applied onto each skin area. In the following, fluorescein gel is also referred to as model drug. On areas a, e, and i, the gel was carefully spread with the tip of a pipette. Areas b, f, and $j$ were treated with a careful finger massage for $2 \mathrm{~min}$. Areas c, d, g, h, k, and l were massaged for $2 \mathrm{~min}$ at a $5-\mathrm{Hz}$ frequency with support of the smartphone applica- 


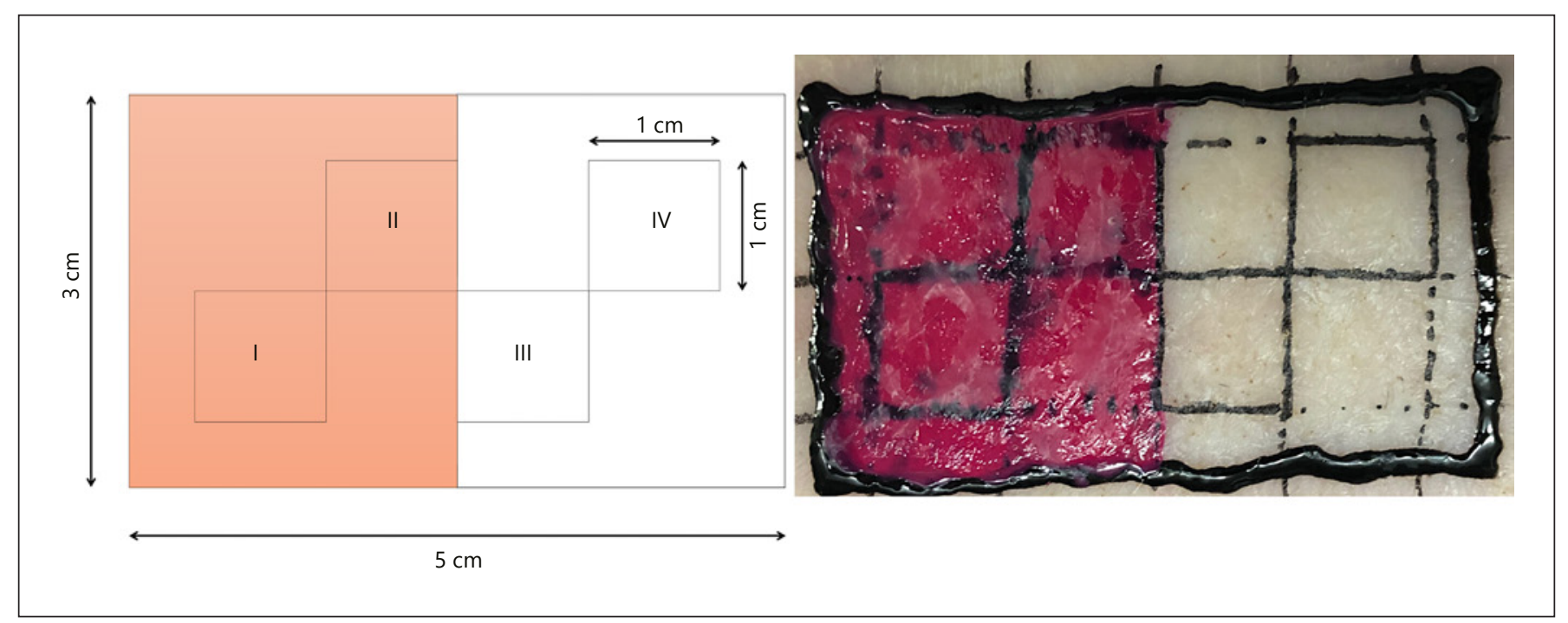

Fig. 2. Graphic illustration and photography of the $3 \times 5 \mathrm{~cm}$ area with four $1 \times 1 \mathrm{~cm}$ areas to investigate the impact on substance penetration of a fully covered penetration area (orange) adjacent to a non-covered area (white).

tion "Pro Metronome" (EUMLab Xainin Technology GmbH, Berlin, Germany). While in areas $\mathrm{c}, \mathrm{g}$, and $\mathrm{k}$ the massage was realized by fingertips, in areas $\mathrm{d}, \mathrm{h}$, and $\mathrm{l}$, a massage device (NOVAFON pro, NOVAFON GmbH, Weinstadt, Germany) was used.

After 2 min, including the massage time, skin biopsies of $0.5 \times$ $0.5 \mathrm{~cm}$ were removed from all skin areas by means of a scalpel, transferred to cryotubes, and shock-frozen in liquid nitrogen. The samples were stored at $-20^{\circ} \mathrm{C}$ until further use. After embedding the skin biopsies in cryomedium (Tissue Freezing Medium, Leica Biosystems Richmond Inc., Richmond, IL, USA), vertical sections of $10 \mu \mathrm{m}$ in thickness were prepared by means of a cryotome (Microm Cryo-Star HM 560, MICROM International GmbH, Walldorf, Germany) and transferred onto microscope slides. The cryosections were investigated by laser scanning microscopy (LSM 700, Carl Zeiss AG, Oberkochen, Germany) at a magnification factor of 10 in both transmission mode and fluorescence mode. By exciting the probe at a wavelength of $488 \mathrm{~nm}$ and applying a laser power of $1 \mathrm{~mW}$, it was possible to visualize the fluorescent dye with a detection range of the emission from 492 to $700 \mathrm{~nm}$ on the skin and in the hair follicles. This enabled the assessment whether and to what extent the follicular closure could efficiently prevent the follicular penetration of the fluorescein gel.

In setup A2, three skin areas of $2.5 \times 2.5 \mathrm{~cm}$ were prepared from three different pigs. Here, the complete skin areas were treated with either standard nail varnish (area $\mathrm{m}$ ) or solvent-free nail varnish (area $\mathrm{n}$ ). The varnish was applied by syringe. One skin area remained untreated and served as control. After a drying time of $1 \mathrm{~h}, 10 \mu \mathrm{L} / \mathrm{cm}^{2}$ of a caffeine gel ( $2 \%$ caffeine [Sigma-Aldrich, Steinheim, Germany] in $\mathrm{H}_{2} \mathrm{O}$ and $7 \%$ hydroxyethyl cellulose, prepared $24 \mathrm{~h}$ before application) were spread onto the center $1 \times 1 \mathrm{~cm}$ of the larger area with the tip of a pipette. For later analysis, only this center $1 \times 1 \mathrm{~cm}$ area was investigated. This setup ensures that no caffeine contacts the tissue without having to pass the varnish barrier.

After an incubation time of $20 \mathrm{~h}$, the unabsorbed test substance and the first layers of the stratum corneum were removed by tak- ing five tape strips (tesafilm ${ }^{\circledR} 4129$, Beiersdorf, Hamburg, Germany). The area of interest was cut out of the tape and placed in a reaction vessel with $1 \mathrm{~mL}$ phosphate-buffered saline (PBS; VWR LIVE SCIENCE; pH 7.4; sterile). Furthermore, the lower layers of the stratum corneum and the epidermis were separated from the dermis and the subcutaneous tissue. This separation step was performed by removing the skin piece from the cartilage with a scalpel and placing it, dermis facing down, on a $60^{\circ} \mathrm{C}$ heating plate for 60 $s$ [22]. Afterwards the epidermis and the dermis were separated by using tweezers. The epidermis was placed in a reaction vessel with $500 \mu \mathrm{L}$ PBS. The large amount of dermal tissue was split into two reaction vessels. To each one, $1 \mathrm{~mL}$ of PBS was added. Homogenization was carried out with a TissueLyser II (QIAGEN, The Netherlands) in a pre-cooled setup with 5 - $\mathrm{mm}$ steel beads. The epidermis and the supernatant were homogenized using 1 cycle of $30 \mathrm{~Hz}$ and $10 \mathrm{~min}$. The dermis was homogenized by applying this cycle twice. All samples were placed into an ultrasound bath (SONOREX SUPER RK 102 H, BANDELIN electronic GmbH \& Co. KG, Berlin, Germany) for $10 \mathrm{~min}$ and were centrifuged (UNIVERSAL 320 R, Hettich AG, Bäch, Switzerland, program: 11,200 rpm; $10 \mathrm{~min}$; $r=85 ; 4^{\circ} \mathrm{C}$ ). The caffeine solution was separated and pipetted into a new reaction vessel. The samples were filtered afterwards with a Sterivex ${ }^{\mathrm{TM}}$ filter unit (Millipore, USA) and the quantification of caffeine was carried out by reverse-phase high-performance liquid chromatography (HPLC), as described below.

Study Design B: Investigation of the Influence of Follicular Closing Material on the Penetration of Adjacent Skin Areas

In study design $\mathrm{B}$, only the standard nail varnish was tested, as study design A revealed that the solvent-free nail varnish is not able to close the hair follicles effectively.

For this experiment, one skin area of $3 \times 5 \mathrm{~cm}$ was marked. Afterwards, one half of the skin area was completely covered with the standard nail varnish, as demonstrated in Figure 2. After 1 h, 10 $\mu \mathrm{L} / \mathrm{cm}^{2}$ of the same caffeine gel as was used in the second setup of study design A was applied by the tip of a pipette on the whole skin 
area. In this treated space, four $1 \times 1 \mathrm{~cm}$ areas of interest were investigated: (I) an area which was completely covered by the closure material, (II) an area which was completely covered by the closure material but located at the edge to the uncovered area, (III) an area which was not covered by the closure material but located at the edge to the covered area, and (IV) an area which was not covered by the closure material. After an incubation time of $20 \mathrm{~h}$ for each of these areas, the unabsorbed test material was removed from the skin surface and skin separation, homogenization, and determination of caffeine concentration were performed analogous to study design A.

\section{Determination of Caffeine in Homogenized Skin Extracts by}

HPLC

The caffeine concentration in the homogenized samples was determined using a HPLC VWR-Hitachi ELITE LaChrom system. The quantity of caffeine was detected at a wavelength of $272 \mathrm{~nm}$ with a DAD 1-2450 detection unit and a column temperature of $30^{\circ} \mathrm{C}$. The analytical determination was performed using a Chromolith ${ }^{\circledR}$ Performance RP-18e 100-4.6 mm (Merck, Darmstadt) column as stationary phase. The results were obtained with a flow rate of $2.0 \mathrm{~mL} / \mathrm{min}$ and an isocratic method with a mobile phase of $90 \%$ water (Milli-Q ${ }^{\circledR}$ ) and 10\% acetonitrile (HPLC gradient grade, Merck, Darmstadt).

Prior to the analysis, the samples were mixed and transferred into auto sampler screw micro-vials (VWR) and analyzed with an injection volume of $10-30 \mu \mathrm{L}$ of each sample. The chemical stability was confirmed by analyzing the caffeine samples after 7 days at the latest. The specificity of the HPLC run was controlled with a blank injection for internal standard.

The quantification linearity for caffeine was confirmed by a caffeine standard solution and quality control of $0.5-250.0 \mu \mathrm{g} / \mathrm{mL}$ in the caffeine samples. A graphic linear regression conformation was determined by an $\mathrm{R}^{2}>0.99$. Accuracy and precision of the HPLC run was determined within the acceptance criteria of a variation of less than $2 \%$.

\section{Statistical Analysis}

The calculation of mean values and standard deviations of the penetration as well as corresponding statistical tests (Wilcoxon test) were carried out via SPSS ${ }^{\circledR}$ (IBM $^{\circledR}$ SPSS $^{\circledR}$ Statistics, version 23.0.0.2, IBM $^{\circledR}$ Corporation, New York, NY, USA). Statistical outliers were determined using the "Grubbs test" via Origin (OriginPro, version 2019, Origin Lab Corporation, Northampton, MA, USA). A significance of $p<0.05$ was considered as being significantly different in this study.

\section{Results and Discussion}

\section{Study Design A: Comparison of the Efficacy of a}

Standard Nail Varnish and a Solvent-Free Nail

Varnish for Selective Hair Follicle Closure and

Investigation of the Impact of the Application Protocol

The aim of study design A was to determine an efficient hair follicle closure material that reliably inhibits substances from penetrating into the hair follicles. Teich- mann et al. [10] established a follicular closure method where nail varnish is used to block the follicular penetration pathway. As concerns had been raised that the solvents of nail varnishes might enhance the penetration behavior of substances in adjacent skin areas, the present study compares the efficacy and potential side effects of a standard nail varnish and a solvent-free nail varnish. Moreover, different application techniques of the model drug were evaluated for their potential to destroy an intact follicular closure. A detection of fluorescein gel in the hair follicle below the nail varnish was evaluated as inefficient closure. After spreading the fluorescein gel with the tip of the pipette, the standard nail varnish was able to prevent the model drug from penetrating into the hair follicle (Fig. 3a). No fluorescent signal could be detected in the hair follicle. When the same application technique was used in combination with the solvent-free nail varnish (Fig. 3e), the nail varnish itself was penetrated by the fluorescein gel and could not provide an intact closure. The combination of the standard nail varnish and a careful finger massage (Fig. 3b) resulted in an efficient closure of the hair follicle, while the $5-\mathrm{Hz}$ massage with a finger (Fig. 3c) or massage device (Fig. 3d) destroyed the nail varnish structure.

For each combination of closing material and application protocol of the model drug, similar results were obtained. In Figure 3, laser scanning microscopic images are exemplarily presented showing the efficacy of the standard nail varnish and the solvent-free nail varnish when the fluorescein gel was applied using different techniques.

The results of study design A1 clearly demonstrate that the standard nail varnish was able to prevent the model drug from penetration into the hair follicle. The solventfree nail varnish did not ensure a full blocking of the hair follicles. This could be due to the microscopic structures of the varnishes, which are strongly different, as can be seen in Figure 3, top row. It is obvious that the standard nail varnish has an extremely fine structure, whereas the texture of the solvent-free nail varnish is rather porous. This might be the reason why the fluorescein gel was able to penetrate the solvent-free nail varnish and to fill the pores, which resulted in an inefficient closure independent of the application technique. The absence of certain solvents in the solvent-free formulations could explain this observation. Solvent systems are indispensable for an improved quality of the dried enamel surface [23].

For standard nail varnish, the application protocol of the model drug was demonstrated to have an impact on the efficiency of the follicular closure. Only spreading the model drug with the tip of a pipette or applying a careful 


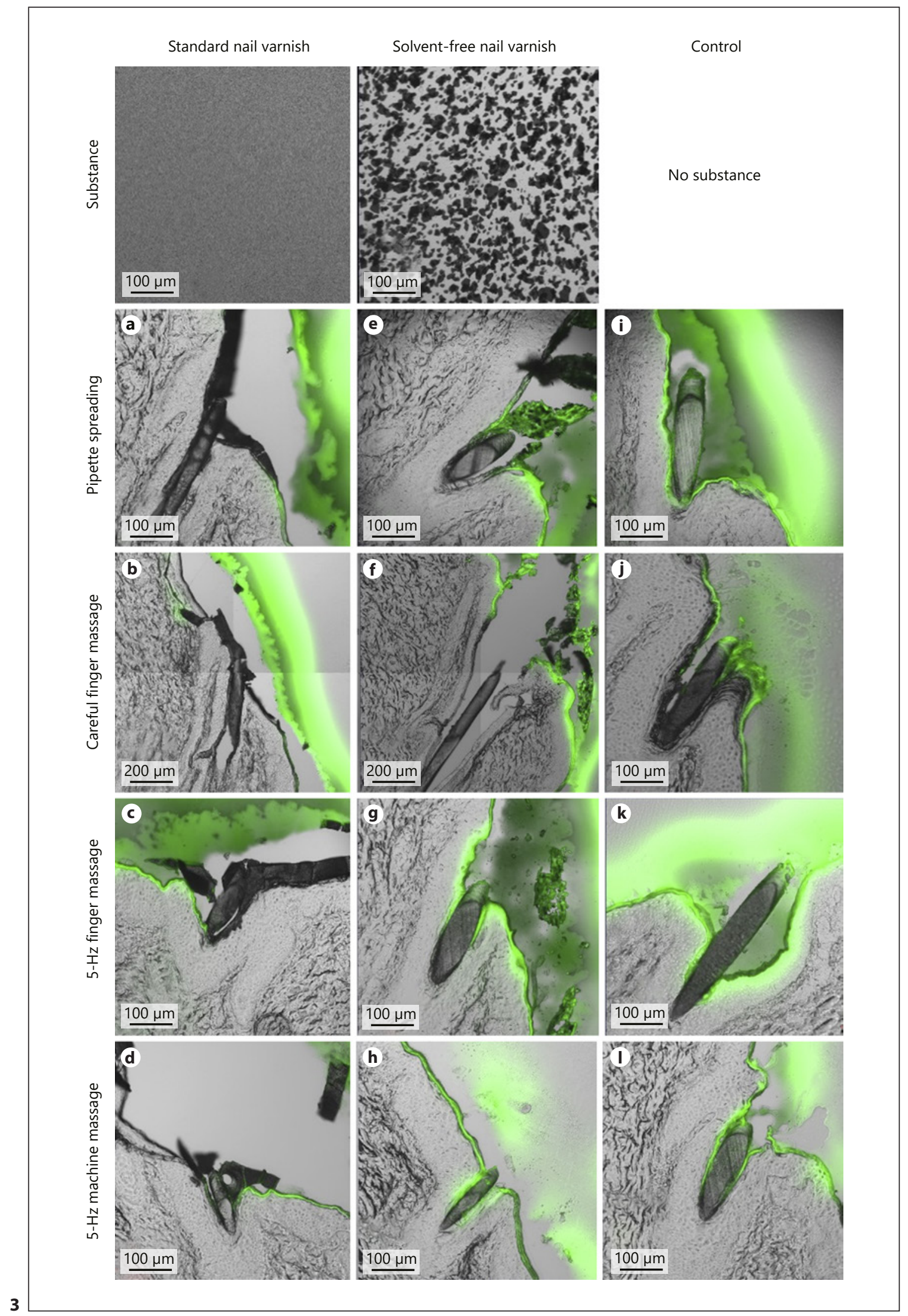

(For legend see next page.)

Skin Pharmacol Physiol 2020;33:117-125 DOI: $10.1159 / 000505839$

Klein et al. 
finger massage keeps the follicular closing intact, stronger mechanical manipulations such as faster finger massage or the utilization of a massage device resulted in a destruction of the follicle closure. In this case, also an enhanced follicular penetration might occur due to capillary forces. Liquids penetrate faster due to higher adhesive forces in the furrows, which are dependent on the surface tension, contact angle, and viscosity at smaller diameters [24].

In the study design using setup A2, one area was completely covered with the closing materials to clarify whether the closure material is able to reliably prevent caffeine penetration also during a longer incubation time.

The results of this experiment support the hypothesis that the standard nail varnish is able to act as an efficient barrier, as no caffeine was detectable in the epidermis and dermis. In contrast, the solvent-free nail varnish was not able to prevent caffeine penetration, as $0.6 \pm 0.1 \%$ of the totally recovered caffeine was found in the tissue, even though the area was covered with the solvent-free varnish.

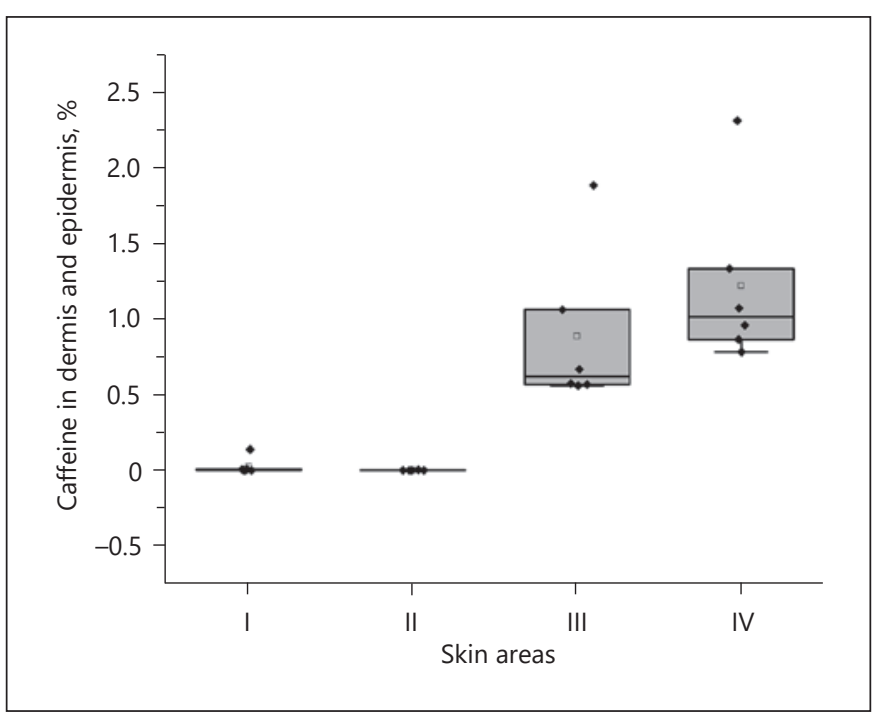

Fig. 4. Boxplot diagram displaying the relative caffeine concentrations in epidermis and dermis in all skin areas I, II, III, and IV. No caffeine concentration was detected in epidermis and dermis of skin areas I and II, which were completely covered with the standard nail varnish. No significant differences were detected between the caffeine penetration rates of skin areas III and IV, which were not covered by the standard nail varnish, but which were at different distances to the covered skin areas $(p=0.138)$.

Fig. 3. Laser scanning microscopic images of hair follicles closed with either a standard nail varnish or a solvent-free nail varnish and unclosed hair follicles (control).

Solvent-Containing Closure Material Can Be Used to Prevent Follicular Penetration
Based on the presented results, it can be assumed that previously published data of the follicular closing technique with standard nail varnish can be considered as reliable. The nail varnish used in the study by Otberg et al. [5] and Blume-Peytavi et al. [25] for follicular penetration studies was comparable to the standard nail varnish utilized in this study. Additionally, the drug formulations under investigation in these studies were applied by careful finger massage; therefore, a disruption of the artificially created barrier has unlikely occurred during their experiments. Besides nail varnish, other researchers also utilized other materials for blocking hair follicles, such as equal amounts of silicone grease and $\alpha$-cyanoacrylate adhesives [26]. This plugging mixture is considered a tool for follicle closure. Several investigations analyzed the follicular penetration pathway successfully on porcine ear skin that was removed from the cartilage and placed on Franz diffusion cells. Indeed, the higher viscosity of the used closure material and the use of a 0.5 - $\mathrm{mm}$-diameter polypropylene stick for the application [27] could lead to a less precise follicular closure, since the closure material does not enter the follicular shunt but rather sits on top of the skin.

It is important to estimate whether certain ingredients of follicular closing material, such as solvents, might have an impact on the penetration of the drug under investigation, which was the aim of study design B.

\section{Study Design B: Investigation of the Influence of}

Follicular Closing Material on the Penetration of Adjacent Skin Areas

Isopropyl alcohol, butyl, and ethyl acetates are known to increase the fluidity due to a formation of a solvation shell around the polar groups, which could lead to a loose packing of the stratum corneum lipid [28] resulting in skin barrier disruption. Therefore, study design B investigated whether nail varnish solvents could possibly have penetration-enhancing effects, e.g., on adjacent skin areas.

The caffeine concentration was determined in skin areas which were sealed completely with the standard nail varnish (I and II) or which were directly next to the sealed area (III) or at a distance of $1 \mathrm{~cm}$ from the sealed area (IV), as illustrated in Figure 2.

In case of a penetration-enhancing effect of the standard nail varnish, an increased concentration of caffeine in area III would be expected. Since area II was completely covered by the standard nail varnish, it was assumed that no caffeine should be detected in this area. However, due to potential effects of lateral diffusion, it 
could not be excluded that area II shows a small caffeine concentration.

The results demonstrate that no caffeine could be detected in areas I and II, which were completely covered by the standard nail varnish. Lateral diffusion effects into area II were not observed. Moreover, the penetration rates of caffeine detected in areas III and IV were statistically of no significant difference $(p>0.05)$. The Grubb's test revealed one statistically relevant outlier each, in three of the groups displayed in Figure 4, but they were not considered when testing differences in caffeine concentrations with the Wilcoxon test. Figure 4 shows the caffeine concentrations detected in the epidermis and dermis of each skin area (I-IV).

The experiments of study design B show that when the area is completely covered with the standard nail varnish, caffeine can be found neither in the epidermis nor in the dermis. Therefore, it can be concluded that the application of the standard nail varnish leads to a secure blocking of the hair follicles and/or the skin without affecting the penetration behavior in adjacent skin areas. The nail varnish exposure when performing the follicular closing technique is much smaller and of shorter duration than in the setup of Friend et al. [28], which was referred to earlier. Effects on stratum corneum lipids are therefore much less likely. It is therefore understandable why our study does not come to the same conclusion as the cited study.

The experiments presented in this study reveal a $1 \%$ caffeine recovery as percutaneous absorption in the tissue. In most studies, the investigation of ex vivo caffeine penetration is performed by using the Franz diffusion cell. In these studies, the penetration rates of caffeine into the epidermis and dermis of $2-5 \%$ were mostly higher than in the present study $[13,29]$. One possible explanation for this variation could be the method used. For the caffeine extraction, no organic solvent was used. In other studies, the Franz diffusion cell is mostly used for quantitative measurements [13]. This study was performed on intact skin on cartilage. It would be of value to compare results directly with in vivo microdialysis or in vitro Franz cell measurements using the same techniques. Another explanation for this higher value could be explained by a hydration effect which could occur in Franz diffusion cell experiments [30], eventually leading to a loosening of the desmosomes between the corneocytes [31]. Differences in the application time on human ex vivo skin ( $24 \mathrm{~h})$ could also account for this finding. Rubio et al. [32] reported a percutaneous absorption of caffeine into the tissue of $1 \%$ by applying a liquid caffeine formulation. It can be summarized that the achieved recovery in the dermis is comparable to the published data.

The study confirms a benefit in follicle closure techniques with a solvent-containing nail varnish. Solvent systems containing polyvinyl acetates, acetate copolymers, and multiple acrylate-type polymers assure an acceptable product viscosity, fast drying time, and a quality of the dried enamel to ensure a non-bubbling nail enamel composition with a good water and oil resistance and a strong coating [23]. So far, the follicular closing technique by varnish has always been performed with products that were comparable to the standard nail varnish used in the present study. The results of previous studies demonstrated that the penetration is affected by excluding the follicular pathway. Otberg et al. [5] showed that by blocking the hair follicle by the varnish in vivo, caffeine becomes bioavailable later and at a smaller amount than in the control area. The same effect was monitored in further studies using the here investigated technique in vivo $[25]$ as well as in vitro $[5,33]$.

\section{Conclusion}

This study clearly demonstrates that a standard nail varnish in combination with a gentle application technique of the model drug is able to prevent follicular penetration successfully and provides a secure follicular closure. Although solvents are a standard ingredient of standard nail varnishes, this ingredient is not expected to cause penetration-enhancing effects in adjacent skin areas. Since comparable solvent-containing nail varnishes have widely been used for evaluating follicular penetration impacts, those studies can be considered to show reliable penetration data and closure of the follicle. The presented study only investigated the closure for the substances caffeine and fluorescein sodium salt. The results might not be transferable to all kinds of APIs.

\section{Acknowledgement}

We would like to thank Mr. Gerald Nusche for providing the porcine ears for the experiments.

\section{Statement of Ethics}

The experiments were authorized by the Commission of Consumer Protection and Agriculture, District Dahme-Spreewald, Germany. 


\section{Disclosure Statement}

The authors have no conflicts of interest to declare.

\section{Funding Sources}

Merck KGaA funded this research.

\section{Author Contributions}

Conception and design of experiments: Anna Lena Klein, Markus Lubda, Alexa Patzelt, Jürgen Lademann, Ingeborg Beckers, Jörg von Hagen, Harald Kolmar. Acquisition of data: Anna Lena Klein, Markus Lubda, Paniz Akbarzadeh Taghavi. Analysis of data: Markus Lubda, Anna Lena Klein, Alexa Patzelt. Interpretation of data: Anna Lena Klein, Markus Lubda, Alexa Patzelt. Drafting the work: Anna Lena Klein, Markus Lubda. Revising the work: Alexa Patzelt, Jürgen Lademann, Ingeborg Beckers, Jörg von Hagen, Harald Kolmar, Paniz Akbarzadeh Taghavi.

\section{References}

1 Maibach H. Dermatological formulations: Percutaneous absorption. By Brian W. Barry. Marcel Dekker, 270 Madison Avenue, New York, NY 10016. 1983. 479 pp. $16 \times 23.5 \mathrm{~cm}$. J Pharm Sci. 1984;73(4):573.

2 Abd E, Yousef SA, Pastore MN, Telaprolu K, Mohammed YH, Namjoshi S, et al. Skin models for the testing of transdermal drugs. Clin Pharmacol. 2016 Oct; $8: 163-76$.

3 Otberg N, Richter H, Schaefer H, Blume-Peytavi U, Sterry W, Lademann J. Variations of hair follicle size and distribution in different body sites. J Invest Dermatol. 2004 Jan;122(1):14-9.

4 Schaefer H, Lademann J. The role of follicular penetration. A differential view. Skin Pharmacol Appl Skin Physiol. 2001;14 Suppl 1: 23-7.

5 Otberg N, Patzelt A, Rasulev U, Hagemeister $\mathrm{T}$, Linscheid M, Sinkgraven R, et al. The role of hair follicles in the percutaneous absorption of caffeine. Br J Clin Pharmacol. 2008 Apr;65(4):488-92.

6 Meidan VM, Bonner MC, Michniak BB. Transfollicular drug delivery - is it a reality? Int J Pharm. 2005 Dec;306(1-2):1-14.

7 Hisoire G, Bucks D. An unexpected finding in percutaneous absorption observed between haired and hairless guinea pig skin. J Pharm Sci. 1997 Mar;86(3):398-400.

8 Honzak L, Šentjurc M, Swartz HM. In vivo EPR of topical delivery of a hydrophilic substance encapsulated in multilamellar liposomes applied to the skin of hairless and normal mice. J Control Release. 2000 May;66(23):221-8.

9 Behl CR, Wittkowsky A, Barrett M, Pierson CL, Flynn GL. Technique for preparing appendage-free skin (scar) on hairless mouse. J Pharm Sci. 1981 Jul;70(7):835-7.

10 Teichmann A, Otberg N, Jacobi U, Sterry W, Lademann J. Follicular penetration: development of a method to block the follicles selectively against the penetration of topically applied substances. Skin Pharmacol Physiol. 2006;19(4):216-23.

11 Liu X, Grice JE, Lademann J, Otberg N, Trauer S, Patzelt A, et al. Hair follicles contribute significantly to penetration through human skin only at times soon after application as a solvent deposited solid in man. Br J Clin Pharmacol. 2011 Nov;72(5):768-74.
12 Horita D, Yoshimoto M, Todo H, Sugibayashi K. Analysis of hair follicle penetration of lidocaine and fluorescein isothiocyanate-dextran $4 \mathrm{kDa}$ using hair follicle-plugging method. Drug Dev Ind Pharm. 2014 Mar;40(3):345-51.

13 Trauer S, Lademann J, Knorr F, Richter H, Liebsch M, Rozycki C, et al. Development of an in vitro modified skin absorption test for the investigation of the follicular penetration pathway of caffeine. Skin Pharmacol Physiol. 2010;23(6):320-7.

14 Verma DD, Fahr A. Synergistic penetration enhancement effect of ethanol and phospholipids on the topical delivery of cyclosporin A. J Control Release. 2004 May;97(1):55-66.

15 Denda M, Sato J, Masuda Y, Tsuchiya T, Koyama J, Kuramoto M, et al. Exposure to a dry environment enhances epidermal permeability barrier function. J Invest Dermatol. 1998 Nov;111(5):858-63.

16 Gee CM, Nicolazzo JA, Watkinson AC, Finnin BC. Assessment of the lateral diffusion and penetration of topically applied drugs in humans using a novel concentric tape stripping design. Pharm Res. 2012 Aug;29(8):2035-46.

17 Chapman SJ, Walsh A. Desmosomes, corneosomes and desquamation. An ultrastructural study of adult pig epidermis. Arch Dermatol Res. 1990;282(5):304-10.

18 Herbig ME, Houdek P, Gorissen S, ZornKruppa M, Wladykowski E, Volksdorf T, et al. A custom tailored model to investigate skin penetration in porcine skin and its comparison with human skin. Eur J Pharm Biopharm. 2015 Sep;95 Pt A:99-109.

19 Franz TJ, Lehman PA, Raney SG. Use of excised human skin to assess the bioequivalence of topical products. Skin Pharmacol Physiol. 2009;22(5):276-86.

20 Stahl J, Niedorf F, Wohlert M, Kietzmann M. The in vitro use of the hair follicle closure technique to study the follicular and percutaneous permeation of topically applied drugs. Altern Lab Anim. 2012 Mar;40(1):51-7.

21 OECD. Test No. 427: Skin Absorption: In Vivo Method. 2004.

22 Baumberger JP, Suntzeff V, Cowdry E. Methods for the separation of epidermis from dermis and some physiologic and chemical properties of isolated epidermis. J Natl Cancer Inst. 1942;2(5):413-23.
23 Graves GG, Jacks TC. Fast drying nail enamel composition and method. Google Patents; 1999.

24 Kwon S, Kim B, An S, Lee W, Kwak HY, Jhe W. Adhesive force measurement of steadystate water nano-meniscus: effective surface tension at nanoscale. Sci Rep. 2018 May;8(1): 8462.

25 Blume-Peytavi U, Massoudy L, Patzelt A, Lademann J, Dietz E, Rasulev U, et al. Follicular and percutaneous penetration pathways of topically applied minoxidil foam. Eur J Pharm Biopharm. 2010 Nov;76(3):450-3.

26 Horita D, Yoshimoto M, Todo H, Sugibayashi K. Analysis of hair follicle penetration of lidocaine and fluorescein isothiocyanate-dextran $4 \mathrm{kDa}$ using hair follicle-plugging method. Drug Dev Ind Pharm. 2014 Mar;40(3):34551.

27 Fadli AJ. Siti Nur S. J., Samer A. D., S. Budiasih, Jawad A., M. Kaleemullah. Determination of Permeation Pathway of Gentamicin into Pig Ear Skin. International Research Journal of Pharmacy. 2015;6(3):183-90.

28 Friend D, Catz P, Heller J. Simple alkyl esters as skin permeation enhancers. J Control Release. 1989;9(1):33-41.

29 Gerstel D, Jacques-Jamin C, Schepky A, Cubberley R, Eilstein J, Grégoire S, et al. Comparison of protocols for measuring cosmetic ingredient distribution in human and pig skin. Toxicol In Vitro. 2016 Aug;34:153-60.

30 Van Hal DA, Jeremiasse E, Junginger HE, Spies F, Bouwstra JA. Structure of fully hydrated human stratum corneum: a freezefracture electron microscopy study. J Invest Dermatol. 1996 Jan;106(1):89-95.

31 Schaefer H, Redelmeier TE, Lademann J. Skin penetration. Contact dermatitis. Springer; 2011. pp. 215-27.

32 Rubio L, Alonso C, Coderch L, Parra JL, Martí M, Cebrián J, et al. Skin Delivery of Caffeine Contained in Biofunctional Textiles. Text Res J. 2010;80(12):1214-21.

33 Trauer S, Patzelt A, Otberg N, Knorr F, Rozycki C, Balizs G, et al. Permeation of topically applied caffeine through human skin - a comparison of in vivo and in vitro data. $\mathrm{Br} J$ Clin Pharmacol. 2009 Aug;68(2):181-6.
Solvent-Containing Closure Material Can Be Used to Prevent Follicular Penetration
Skin Pharmacol Physiol 2020;33:117-125 DOI: $10.1159 / 000505839$ 